\title{
BMJ Open Diagnostic strategies in general practice and the emergency department: a comparative qualitative analysis
}

\author{
Stefan Bösner, ${ }^{1}$ Jamal Abushi, ${ }^{1}$ Markus Feufel, ${ }^{2}$ Norbert Donner-Banzhoff ${ }^{1}$
}

To cite: Bösner S, Abushi J, Feufel M, et al. Diagnostic strategies in general practice and the emergency department: a comparative qualitative analysis. BMJ Open 2019;9:e026222. doi:10.1136/ bmjopen-2018-026222

- Prepublication history and additional material for this paper are available online. To view these files, please visit the journal online (http://dx.doi org/10.1136/bmjopen-2018026222).

Received 22 August 2018 Revised 19 February 2019 Accepted 4 April 2019

Check for updates

(c) Author(s) (or their employer(s)) 2019. Re-use permitted under CC BY-NC. No commercial re-use. See rights and permissions. Published by BMJ.

${ }^{1}$ Department of General Practice/Family Medicine, Philipps University of Marburg, Marburg, Germany ${ }^{2}$ Department of Psychology and Ergonomics, Division of Ergonomics, Technische Universitat Berlin, Berlin, Germany

Correspondence to

Dr Stefan Bösner;

boesner@staff.uni-marburg.de

\section{ABSTRACT}

Objective We sought to explore differences and commonalities between diagnostic strategies used by clinicians in general practice and the emergency department.

Design Qualitative study.

Settings We videotaped 282 consultations of 12 general practitioners (GPs) in Germany, irrespective of presenting complaint or final diagnosis. Reflective interviews were performed after each consultation. In addition, 171 consultations of 16 emergency physicians (EPS) based at two tertiary care hospitals in the Midwest of the USA were observed, and their conversations recorded. Recordings of consultations and GP interviews were transcribed verbatim and analysed using a coding system that was based on published literature and systematically checked for reliability.

Results EPs more often considered acute and severe conditions, even if pretest probabilities were low. In contrast, GPs more often involved their patients in the decision-making process and provided assurance concerning their complaints. To focus their workup, EPs used a more directive style of interviewing including a high proportion of routine questions and rarely used open questions or active listening.

Conclusions Strategies used by physicians in both settings seem to be well adapted to their respective environments. Whereas the physician-led diagnostic process in the emergency department is well suited to rule out life-threating disease, diagnosis and appropriate treatment of everyday problems may require a more patient-centred style.

\section{INTRODUCTION}

Making a diagnosis is perhaps the most important task the physician has to fulfil. Getting to know the cause of their problems and associated prognostic and therapeutic implications is the foremost reason why patients consult physicians.

There are two settings where the diagnostic task poses a particular challenge. General practice and hospital emergency departments (EDs) are at the entrance to the healthcare system. In both settings, unselected patients present problems ranging from benign and/or undifferentiated conditions

\section{Strengths and limitations of this study}

Large patient samples representative of the respective environment.

- Investigation of decision-making based on real patient-physician encounters.

- GP sample biased towards experienced practitioners and those actively involved in medical education.

- Video recordings, observations and interviews presumably interfered with GPs' and EPs' behaviour and the accounts of their reasoning.

to life-threatening emergencies. Both settings are highly relevant for the healthcare system as a whole, because clinicians in both settings act as gatekeepers and indirectly control access to other specialties by the referral of patients.

While general practice and hospital EDs are both a point of entry to the healthcare system, there are also important differences between these settings. Patients interpreting their symptoms as severe or even life-threating are encouraged to access hospital EDs. Here, advanced diagnostic and therapeutic technologies are available, whereas general practice is limited in this regard. While the former limits itself to managing single illness episodes, the latter prides itself for providing continuous and comprehensive care. In most industrialised countries, specialties of general practice and of emergency care have developed their own distinct traditions of clinical strategies, teaching and research.

Although diagnostic reasoning and decision-making by clinicians have been studied in both settings, ${ }^{1-5}$ a direct comparison has not yet been undertaken. We sought to explore differences and commonalities between diagnostic strategies used by clinicians in both settings. In addition, we aimed to explore how physicians in both settings define and understand their relationship with the patient and their role as decision makers within the wider healthcare system. 


\section{METHODS}

Two existing datasets of studies investigating general practitioners' (GPs) and hospital emergency physicians' diagnostic reasoning provided a unique opportunity to meet the objectives formulated above. The methods of both studies have been described in detail in previous publications. $^{45}$

A checklist regarding standards for reporting qualitative research is added as a online supplementary file.

\section{Settings, samples and data collection \\ Emergency department}

Data were collected between 2008 and 2009. Twelve emergency physicians (EP) were shadowed during their 10-hour shifts at the ED of two teaching hospitals in the Midwest of the USA. Two observers (two researchers with Masters' degrees in psychology) independently observed and documented EP' actions and verbal interactions with patients, relatives and colleagues using shorthand. All participants agreed to be part of the study. Unconscious patients and patients in need of emergency treatment were not observed. Particular interest was on the exchanges between residents and their supervising attending physicians as they naturally provided insight into their reasoning and decision-making strategies without requiring explicit prompts. Both observers followed a strict protocol of silent observation to keep their presence as unobtrusive as possible. They compared their records regularly and corrected possible discrepancies in the data collection process.

\section{General practice}

Data were acquired between 2010 and 2012. Twelve fulltime GPs in the Marburg-Biedenkopf district of Hessen, Germany, were asked to take part in the study and all agreed. They had at least 10 years clinical experience in practice and were active teachers associated with University of Marburg Medical School. GPs were videotaped on three different occasions during consultations with patients who gave their consent in advance. GPs recruited patients for the study, regardless of their complaint. The only exception were purely administrative visits with no diagnostic implications. Participating GPs informed each patient about the study and asked for written consent to have their consultation video recorded. GPs were instructed not to address the patient's presenting complaint(s) at this stage. After consent was obtained, consultations proceeded as usual. Sessions were scheduled so that after each consultation, GPs had sufficient time for a semistructured interview to explain their diagnostic reasoning. Interviews were conducted by an experienced GP researcher and also video recorded. GPs were asked to elaborate on their first impression and previous knowledge of each patient, diagnostic hypotheses they considered and diagnostic data they gathered.

Both the written protocols for the ED and the video material were transcribed verbatim and coded by two researchers with MAXQDA software for qualitative data analysis. ${ }^{6}$ We considered a consultation as containing a diagnostic episode if the patient brought up a new complaint, which resulted in some kind of data collection by the physician, such as taking a history, conducting a physical examination and so on.

\section{Data analysis}

Drawing on previously published work, ${ }^{2-9}$ we developed a coding tree comprising categories describing EP' and GPs' diagnostic reasoning strategies and data collection behaviour (the coding tree is available on request). Extensive discussion in our group and several iterative loops of coding and modification of the coding tree resulted in operational definitions for the identified categories (see below).

We used a randomly selected subsample (59 consultations of both the GP and EP data) for quantifying the number of cues physicians collected using each diagnostic strategy. We defined as cue any piece of (medically) relevant information either spontaneously verbalised by the patient or specifically asked by the physician. This included also non-verbal information derived from observation, the physical examination or diagnostic tests.

To evaluate the reliability of the codings, a subsample of 31 consultations was coded by two independent researchers. The resulting codes focused not only on diagnostic strategies but also on the diagnostic cues that participating physicians obtained using these strategies. To determine inter-rater reliability (IRR), Holsti's coefficient of reliability was calculated. ${ }^{10}$ Data analysis followed the concept of qualitative content analysis. ${ }^{11}$

\section{Definitions of relevant codes}

Inductive foraging

A phase most often (but not necessarily) at the beginning of a consultation, in which the patient is encouraged to freely explain the reason of his visit and associated concerns. ${ }^{9}$ This strategy is mostly prompted by an open question asked by the physician.

\section{Triggered routine}

A sequence of questions either specific to an organ system, a specific symptomatic or pathomechanism. Questions are triggered by a symptom or finding and aim to further explore a clinical area.

\section{Deductive testing}

The physician has a specific hypothesis (eg, disease) in mind, and asks one or more targeted questions to either dismiss or substantiate his hypothesis. Deductive testing differs from a triggered routine, as it is clearly hypothesis driven.

\section{Descriptive question}

Questions that invite the patient to further describe his symptoms or bodily functions, for example, in terms of exact location, colour, quality and so on. 
Checklist routine

A large number of consecutive questions covering a broad diagnostic spectrum, often containing questions regarding risk factors and very common symptoms or diseases. These items are often only remotely related to the presenting complaint.

\section{Pattern failure}

Any detail or information that does not fit the overall picture of the patient, or points to a general inconsistency within the case.

\section{Probabilistic reasoning}

Considerations of the statistical frequency of occurrence or likelihood are brought up when deliberating a diagnosis.

\section{Spot diagnosis}

A (most often visually) perceivable feature, symptom or combination thereof immediately leading to a diagnosis.

Self-labelling

The patient or a family member mention or suggest a diagnosis.

\section{Reassurance}

Providing information regarding the benign nature of symptoms or findings, allaying associated concerns and fears.

\section{Data protection and ethics}

The text material used in this study was anonymised. All physicians and patients gave their consent prior to observation.

\section{Patient and public involvement}

Patients or public were not involved in study design, recruitment or dissemination of results.

\section{RESULTS}

\section{Participating clinicians and patients}

The ED sample consisted of 171 patients, compared with 134 patients where a diagnostic process took place (out of 282 videotapes consultations) in the general practice sample. Patients in both samples were similar in distribution of gender and age. There were 16 different EP, compared with 12 GPs. The EP mostly consisted of young residents in training who were supervised by more experienced attending physicians, whereas the GP sample consisted of more experienced physicians only. GPs had on average practiced for 21 years. Of the EP, four were in their first year of residency, five were in their second year, six in their third year and one held the status of an attending. The clinical experience of the supervising attending physicians ranged from 5 to 31 years, with a mean of 13 years (for details, see table 1). IRR of coding was determined based on the codes most essential for analysis of diagnostic strategies (listed in table 2) and ranged between $77 \%$ and $79 \%$. IRR for all cues coded
Table 1 Patient, general practitioner (GP) and emergency physician (EP) characteristics

\begin{tabular}{|c|c|c|}
\hline & $\begin{array}{l}\text { No of patients } \\
\text { seen by EPs } \\
\text { (percentage of all } \\
171 \text { patients) }\end{array}$ & $\begin{array}{l}\text { No of patients } \\
\text { seen by GPs } \\
\text { (percentage of all } \\
134 \text { patients) }\end{array}$ \\
\hline \multicolumn{3}{|l|}{ Gender } \\
\hline Female & $104(61)$ & 85 (63) \\
\hline Male & 65 (38) & 49 (37) \\
\hline Unknown & $2(1)$ & $0(0)$ \\
\hline \multirow{2}{*}{$\begin{array}{l}\text { Average age } \\
\text { (in years) }\end{array}$} & 52.3 & 48.5 \\
\hline & $\begin{array}{l}\text { No of EPs } \\
\text { (percentage of all } \\
16 \text { physicians) }\end{array}$ & $\begin{array}{l}\text { No of GPs } \\
\text { (percentage of all } \\
12 \text { physicians) }\end{array}$ \\
\hline \multicolumn{3}{|l|}{ Gender } \\
\hline Female & $9(56)$ & $5(42)$ \\
\hline \multirow[t]{2}{*}{ Male } & $7(44)$ & $7(58)$ \\
\hline & EPs & GPs \\
\hline $\begin{array}{l}\text { Mean clinical } \\
\text { experience (in years) }\end{array}$ & $\begin{array}{l}\text { Residents: } 2 \\
\text { Attendings: } 13\end{array}$ & 21 \\
\hline
\end{tabular}

was $78.9 \%$ (for details, see table 2). We first report how physicians in both settings define their professional role as decision makers within the wider healthcare system and how they tend to relate to their patients. Then, we elaborate the diagnostic strategies observed in the ED versus GP setting.

\section{Professional roles and patient reassurance Professional roles}

When commenting on their role, EPs present themselves as 'distributionists' rather than diagnosticians. They see their primary task in picking up hints for acute and severe or even life-threatening disease and making sure the patient is admitted to the appropriate department of the hospital or sent home with follow-up. In their view, making a positive diagnosis is not a priority.

RES: We look at emergencies here. If we can't find anything, you'll need good follow-up or to be admitted to answer those questions for you (ED; Res.04, Pt.06).

Att. to RES: Get an ultra-sound to check for a deep blood-clot. I do not worry about the abscess. You

Table 2 Inter-rater reliability for the four most important codes

\begin{tabular}{ll} 
Code & $\begin{array}{l}\text { Coefficient of } \\
\text { reliability (\%) }\end{array}$ \\
\hline Inductive foraging & $\mathrm{R}=77.8$ \\
Triggered routine & $\mathrm{R}=76.9$ \\
Deductive testing & $\mathrm{R}=76.5$ \\
Descriptive question & $\mathrm{R}=79.2$ \\
\hline
\end{tabular}


got to think: 'What's going to kill him!' I guarantee there's a superficial clot, [but] a deep clot, that's what we are going to be judged by! (ED; Res.15, Pt.13).

Pt: I don't like the diagnosis Fibromyalgia, because you put a name on my pain that makes no sense. RES: I don't know, I didn't diagnose it...we don't diagnose in the emergency department! (ED; Res.05, Pt.07).

Patient wants a diagnosis (is curious, not insistent). Attending says that isn't what the emergency department is really set up for. [...] (ED; Att.16, Pt.18).

Contrary to the EPs, GPs often expressed a strong bond with their patients, including the desire to diagnose and treat their patients.

GP: [...] And it is expected of us to have an idea how to approach this, and I try to treat a lot on my own. Of course, I could say: 'Hey, there is pruritus, there is a rash, I'll send you to the dermatologist right away.' But I just don't want to do that (GP.03, Pt.04).

In contrast to EPs who usually only see and treat 'single episodes' of disease, continuity of care played a central role in GPs' professional understanding.

GP: $[\ldots]$ This is a patient who I know to have many worries. Every time I see him, I look at him: Has he become even thinner? Where is his journey taking him? (GP.07, Pt.18).

In addition, GPs see themselves as caring for acute and chronic disease, covering a wide range of issues ranging from biomedical to psychosocial.

GP: That's how it is, how it developed after some time. That you have some common ground, you meet them in different places, know something about them, for example about their jobs. I also often know the family. Because I'm interested in that, and because I find this important as a general practitioner. I want to know the person sitting in front of me. I'm not only here for diseases and their treatment (GP.03, Pt.02).

GP: She's a very anxious patient. Today, not too much, but I also addressed this issue. She has carcinophobia, feeling unwell or being sick cause her to fear a severe reason for that. [...] She's afflicted with fear (GP.11, Pt.23).

\section{Patient reassurance}

Reassurance was present in $18 \%$ of the ED consultations, while it was used by GPs in $64 \%$ of the encounters. There were typical differences between the two professional groups. GPs use words of reassurance mostly to address and possibly relieve patients' fears about a certain diagnosis or personal worries. EPs communicate their general impression to the patient, especially when they consider hospital admission unnecessary and plan to discharge their patient.

GP: Well, there are some extrasystoles. I will look into what kind they are. But they are actually as harmless as they have always been, right? The lung is free [of pathological findings], so there is nothing bad there. Is it like back then; Is it like before your eightieth birthday, when you thought: "I will never turn eighty", but then easily went past eighty? Is it like that? Pt.: It is somewhat like that (laughs) (GP.09, Pt.10).

RES: I think with how well you're looking, [opioid pain medication] should work for you. You're looking pretty well. But of course, come back if necessary, we are happy to have you here and take good care of you. Pt.: Can I have some more water and try if I can keep down the nausea meds? RES: Sure, you should have it in little sips. Pt.: If it works here, it should work at home, too. RES: You should do pretty well, you look good (ED; Res.02, Pt.02).

\section{Diagnostic strategies}

We describe the observed patterns for each coded diagnostic strategy in turn. For a summary of frequencies of the diagnostic strategies in the ED versus GP setting, see table 3 .

\section{Inductive foraging}

Inductive foraging was almost exclusively used at the beginning of a consultation. While GPs used it in the majority of cases (91\% of all consultations), EPs utilised this strategy with only a third of their patients $(33 \%$ of all consultations). For GPs, this phase of the diagnostic process generated on average $26.5 \%$ of diagnostic cues, while in the ED only $4 \%$ of cues were obtained with inductive foraging. In order to initiate inductive foraging, GPs used open questions like 'What leads you to me?', 'What can I do for you?' or 'What seems to be the problem?'.

Table 3 Frequencies of the four most important diagnostic strategies and their relative contribution to the number of all cues generated

\begin{tabular}{lll}
\hline Strategy used & $\begin{array}{l}\text { Emergency department } \\
\text { (\% of all 171 consultations) }\end{array}$ & $\begin{array}{l}\text { General practitioners } \\
\text { (\% of all 134 consultations) }\end{array}$ \\
\hline Inductive foraging (\% of all cues generated) & $33 \%(4)$ & $91 \%(26.5)$ \\
\hline Triggered routines (\% of all cues generated) & $50 \%(26)$ & $45 \%(10)$ \\
\hline Deductive testing (\% of all cues generated) & $28 \%(5)$ & $46 \%(10)$ \\
Descriptive questions (\% of all cues generated) & $21 \%(4)$ & $89 \%(19)$ \\
\hline
\end{tabular}


In the minority of cases, in which GPs did not use inductive foraging, there often was pre-established information about the reason of the patient's visit. In the ED, patients had often already been seen by another health professional, such as a triage nurse. As a result, relevant information had been obtained from the patient prior to the consultation with the physician, reducing the need to engage in inductive foraging.

$\mathrm{RN}$ (registered nurse) in the ED brings chart for a new patient: Shortness of breath, history of asthma. RN called respiratory already (ED; Res.09, Pt.02).

\section{Spot diagnosis}

Spot diagnosis was more common in the GP setting, where it comprised $13 \%$ of cases, compared with only $5 \%$ in the ED.

Att. says it's called Paronychia and explains that it's an infection around the nail (ED; Att. 16, Pt.01).

Pt: Good morning. GP: So, what is it that you have? Oh. Yeah, you have shingles, right? (GP.09, Pt.04).

\section{Triggered routines}

GPs used triggered routines, among other types of directed questions, typically to continue and focus the investigation based on the insights gained with inductive foraging. In the ED preacquired information was often available so that inductive foraging was not necessary. Instead, EPs tended to pick up on the most significant piece of the preacquired information, often the presenting complaint from the triage sheet, and used it as a trigger for further routine questions.

Triggered routines were utilised in $50 \%$ of consultations in the ED, and in $45 \%$ of the cases seen by GPs. The number of cues this strategy yielded differed in the ED compared with the GP setting, with $26 \%$ of all cues per consultation coming from triggered routines in the ED, opposed to $10 \%$ on the GPs' side. Also, the content of triggered routines differed. In the ED, the most frequent categories were neurological, cardiovascular and gastrointestinal symptoms, while GPs used most triggered routines in conjunction with infections of the upper respiratory tract.

\section{Descriptive questions and deductive testing}

GPs used descriptive questions in $89 \%$ of their consultations and they contributed $19 \%$ of all cues. EPs used them in $21 \%$ of their cases and $4 \%$ of cues were generated that way. Deductive testing occurred in $28 \%$ of consultations in the ED and in $46 \%$ of the GP consultations. It contributed $5 \%(\mathrm{ED})$ and $10 \%(\mathrm{GP})$ of all cues on average in the respective subsample.

\section{Checklist routines}

Checklist routines were unique to the ED, where they were used in $19 \%$ of all consultations and generated on average $9.5 \%$ of all cues in a given diagnostic episode.
RES: Nausea, vomiting, dizziness? Pt: No; RES: Belly pain? Pt.: No. RES: Bloody stool? Pt.: No RES: Chills? Pt.: No RES: Sore throat? Pt.: Yes. RES: For how long? Patient has bad allergies; RES: Ahh, me too! Other medical history? Pt.: Antiphospholipid syndrome. [...] Patient has diabetes; RES: High blood pressure? No; Is there a chance you are pregnant? No; Pt.: [My] last period was 2 weeks ago; RES: Was it normal? Pt: Heavy. RES: When was it that they last checked your iodine? Pt.: Last Friday, it was 3.0. RES: Any allergies? Pt.: Morphine, Toradol. RES: Are you a smoker? Pt: No. RES: Alcohol/drugs? Pt: No. RES: Do you have a history of blood clots? Pt: On my dad's side of the family. RES: Surgeries? Pt.: DNC [dilation of the cervix and curettage of the uterus] two times (ED; Res.05, Pt.03).

\section{Pattern failure}

Pattern failure played a role for diagnostic reasoning in 9\% of the ED cases and in $8 \%$ of the cases seen by GPs. As EPs were not familiar with their patients, pattern failure predominantly related to the credibility of the patient's story.

RES: She's an odd duck...And she asked me how old I was. You don't ask that if you are in pain. She had five surgeries in the last couple of months (ED; Res.05, Pt.07).

\section{Probabilistic reasoning}

Overall, $30 \%$ of the GP cases and $4 \%$ of the cases in the ED contained probabilistic reasoning. GPs used probabilities on many occasions to explain their reasoning in depth, both to patients and to the interviewer.

GP: My main suspicion is really a prostate problem, well...a benign one I really hope. Uhm, the main concern in this case is also this: 50 percent do have a tumor at that age, if not even more. 70 percent at age 70 , that's roughly the allocation. So it is actually possible that he is fitting the statistics and has something like that (GP.07, Pt.22).

In the cases where EPs used probabilistic reasoning, they did it in a similar fashion.

RES: Pulmonary embolism changes can be seen in 10 percent of the patients in EKG changes. [RES means 10 percent of people with pulmonary embolism have changes in their EKG] She has some of those changes (ED; Res.05, Pt.03).

\section{Self-labelling}

Self-labelling could be observed in $7 \%$ of the ED patients and in $64 \%$ of the patients seen by the GP.

Pt: [...] I was in the garden last week, and then I couldn't even move anymore. But that is probably a 
muscle soreness because of my old spinal disc history (GP.07, Pt.06).

EPs response to self-labelling differed mainly depending on the phase of the diagnostic process in which the suggestion was made. Patients attempted self-labelling most frequently when EPs asked directed questions. In these cases, EPs usually did not respond, presumably to keep their diagnostic focus on acute and severe diseases.

RES: The rhythm looks fine. You have no abdominal pain?-No; Diarrhea?-No; Blood in your stool?No; What meds are you taking? Pt.: Aspirin and something for memory, they checked for Alzheimer's. Daughter: Maybe he had a small stroke. RES: is it [name of medication inaudible]? Pt doesn't know. RES: I will look it up. Do you take anything for hypertension? [...] (ED; Res.01, Pt.06).

\section{DISCUSSION}

Our study shows that although EPs and GPs are both generalists and gatekeepers to the healthcare system, there are marked differences regarding their professional roles, the frequency of use of diagnostic strategies and their interaction with the patient. Each professional group seems to have a very specific set of skills and processes adapted to its respective working environment.

In their diagnostic assessment of patients, EPs tend to focus on acute serious, life-threatening disease and on triage to make sure that the patient is treated at the right spot of the healthcare system. The outcome of their decision is essentially binary. The either admit a patient to the hospital or discharge with follow-up. In their view and actions, they are hardly concerned with diagnosis and therapy above and beyond a focus on life-threatening diseases. Since they assume that the majority of their patients will obtain definite treatment by other healthcare providers, they also reassure their patients less often than GPs. Although this strategy seems rather reductionist at first glance, it certainly is a pragmatic strategy that helps avoid missing rare but potentially life-threatening diseases despite the complexity of the diagnostic task in an uncertain and stressful environment.

GPs, on the other hand, treat the whole range of biomedical and psychosocial problems presented by their patients, including long-term management. They can do so only because they see their patients repeatedly so that they can accumulate knowledge about each patient regarding risk factors, previous disease episodes, treatments, healthcare utilisation behaviour and so on. Although individual consultations may be short in general practice, the advantage of a continuous relationship and the accumulated knowledge of the patient's history helps GPs to quickly focus on the currently most pressing problems and and thus address a much larger variety of problems than during an isolated ED treatment episode, that is, focused on acute and severe diseases.

\section{Diagnostic strategies}

Some strategies were used in both settings with more or less equal frequency, such as triggered routines or pattern failure. For other strategies, however, we found characteristic differences. Inductive foraging and descriptive questions occur more often in general practice and contribute a considerable number of diagnostic cues. Moreover, patients contribute to the diagnostic process by communicating their own explanation in two-thirds of the cases in the GP setting. Checklist routines, on the other hand, are a typical strategy in the ED. At a descriptive level, these differences amount to the patient playing a more active part in general practice, whereas physicians in the ED control the encounter to a much larger degree, for instance, by asking patients mainly closed questions related to potentially life-threatening diseases. This focus may also explain why EPs use probabilistic reasoning less frequently than GPs. Although acute, time critical illness requires immediate action, it is often less probable than more common, benign diseases. ${ }^{5}$

Both inductive foraging and checklist routines are instruments to explore the large problem space generalist physicians are typically confronted with. They differ by who is leading the process. In general practice, at least for a brief moment at the beginning of the consultation, the patient is directing the discussion to relevant problem areas. In the ED, patient input is more controlled by the physician. One explanation for this pattern is that in the latter setting, the initial exploration is often in the hands of nursing staff who pass the resulting information on to the physician.

Pattern failure, that is, noticing a deviation from an assumed pattern or default, plays a role for diagnostic reasoning in both groups but was used differently. For EPs, the most common reference frame is 'the credible patient'. For GPs, pattern failure more often serves an immediate diagnostic purpose. Prior knowledge of the individual patient is crucial for comparing signs and symptoms with an assumed 'normal' state (this does not have to be a healthy or symptom-free state, especially in the chronically ill). ${ }^{9}$

EPs and GPs differ characteristically in their default assumptions about whether life-threatening disease is present or not. EPs always consider life-threatening disease given that their primary objective is to exclude serious etiologies. In general practice, serious diseases occur only rarely. To borrow the wording suggested by Stolper $e t a l^{12}$ : while a 'sense of alarm' is felt by GPs only occasionally and has to be triggered by specific symptoms and/or findings, this seems to be the normal state for ED. This characteristic difference between GPs and EPs reflects the different prevalence of serious disease in their respective settings and is an explanation for the observed differences in diagnostics strategies.

\section{Strengths and limitations}

Our research differs from most published work in the field of medical decision-making in that we investigated 
decision-making based on real patient-physician encounters. In both settings, similar attention was paid during data collection to record the natural, unaltered conversations and actions during the consultations. In order to do so, we included consultations concerning any symptom or disease that patients presented in either the GP or the ED domain. As both samples largely represent their respective patient population, we assume that our observations represent EPs' and GPs' encounters with patients and their actions as naturally as possible to allow the comparison of physicians' diagnostic strategies across the two domains.

There were, on the other hand, important differences regarding methods and settings chosen. The GP sample was purposely biased towards experienced practitioners (practicing medicine for on average 21 years) and those actively involved in medical education. By doing so, we expected to obtain valid in-depth accounts of clinical reasoning. In the ED, we had the same goal but focused on educational interactions between resident and attending physicians to attain it. Whereas the ED sample mainly contained young residents in training supervised by experienced colleagues, the observed GPs were experienced themselves. Thus, resident and attending EPs elaborated their reasoning as part of their natural interaction, and GPs had to be interviewed to have them reflect on their reasoning after each consultation. In addition, the recording technology, that is, video recordings versus written shorthand documentation, yielded different levels of detail in the data which may have influenced data analysis. Also, interference due to an observer in the ED versus a video camera in the GPs' practice may have impacted physicians' responses differently. Differences in training of each group of physicians and differences in the specific 'culture' of the surrounding work environment might have affected our results. That is, our comparison may have been confounded by data collection being undertaken not only in different settings, as intended for the objective of this research, but also in two different countries and healthcare systems (Germany vs the USA). Lastly, our analysis largely focused on the initial history taking and interaction with the patients. We did not analyse the examination techniques or how physicians used the result of test orders and further investigations to revise their reasoning. We did also not include the patients' perspective on the different diagnostic approaches of their physicians.

\section{CONCLUSIONS}

To study decision makers' strategies as an adaptation to the particular structure and challenges of their environment has been a fruitful approach in decision science ${ }^{13} 14$ and approaches to improving work system performance. ${ }^{15}$ If one considers our findings from this descriptive perspective, strategies used by physicians in both settings seem to be well adapted to their respective environments. This descriptive lens should not, however, prevent us from asking whether and how existing approaches may be improved.

The physician-dominated process has been established as the general diagnostic approach in the ED setting to avoid missing rare but life-threatening diseases. However, diagnosis and treatment of everyday complaints may require a more patient-centred style, for instance, in situations when patient input and disease experience is central for physicians to understand the problematic at hand. ${ }^{16}$ When the patient has sufficient time to elaborate on his or her complaints, interpretations and suggestions, ED staff might not need to go through long checklists, which may pose an undue cognitive burden on many patients and lead to answers of questionable validity. Moreover, patients who only passively answer closed questions will likely not point to symptoms or findings not thought of by the enquiring physician. Listening to the patient-for instance, by using inductive foraging-might thus be a strategy that may be used more frequently also in the ED setting. To what extent it may already be used during ED nurse triage, we cannot answer with this study.

The EPs' focus on triage is an effective strategy to avoid missing life-threatening diseases in an environment, that is, marked by high uncertainty, limited resources and time. As a single strategy it is at odds, however, with the clinical needs and expectations of patients seeking help, explanation and therapy for their often minor complaints. Needs related to non-lethal, everyday complaints will likely be more reliably met in GP settings and with the diagnostic strategies we observed there. Thus, our findings support two practical implications. A first implication relates to educational efforts that aim at helping generalist physicians gauge when to rely on EPs' more time-efficient approach to detect critical diseases and when to engage in GPs' more time-intensive but patient-centred diagnostic strategies. The second implication relates to efforts at the policy level that aim at improving access to primary care for patients with everyday complaints while making sure that patients with a sufficiently high likelihood of a life-threatening illness can readily access hospital ED to receive acute treatment. ${ }^{17-19}$

Contributors ND-B, SB and MF designed the research questions. JA did the main data analysis and all authors discussed and revised the findings. SB and ND-B prepared the first draft of the publication and MF and JA revised it.

Funding This research on GPs was supported by the German Research Foundation (DFG) with grant D0 513/2-1 and Gl 170/8-1. There was no funding for the research on EPs.

Competing interests SB, JA, MF and ND-B have no relationships with companies that might have an interest in the submitted work in the previous 3 years; their spouses, partners or children have no financial relationships that may be relevant to the submitted work; and SB, JA, MF and ND-B have no nonfinancial interests that may be relevant to the submitted work. The funder of the study had not influence on study design, collection, analysis and interpretation of data, on the writing of the report or the decision to submit the article for publication. All authors had full access to all of the data in the study and can take responsibility for the integrity of the data and the accuracy of the data analysis. The lead author (SB) affirms that this manuscript is an honest, accurate and transparent account of the study being reported; that no important aspects of the study have been omitted; and that any discrepancies from the study as planned have been explained. 
Patient consent for publication Not required.

Ethics approval The ethic committees of both the involved hospitals (\#08-034) and the medical school/university (SC \#3650) approved the study from which ED data are reported. The ethics committee of the University of Marburg Medical School approved the study from which GP data are derived (file number 39/10).

Provenance and peer review Not commissioned; externally peer reviewed.

Data sharing statement GP sample: video recordings, audio recordings and transcribed interviews are stored at the Department of General Practice, University of Marburg. EP sample: notes and transcripts are stored at Technische Universität Berlin, Department of Psychology and Ergonomics, Division of Ergonomics. Both datasets are currently only available to researchers of the above-mentioned institutions.

Open access This is an open access article distributed in accordance with the Creative Commons Attribution Non Commercial (CC BY-NC 4.0) license, which permits others to distribute, remix, adapt, build upon this work non-commercially, and license their derivative works on different terms, provided the original work is properly cited, appropriate credit is given, any changes made indicated, and the use is non-commercial. See: http://creativecommons.org/licenses/by-nc/4.0/.

\section{REFERENCES}

1. Hampton JR, Harrison MJ, Mitchell JR, et al. Relative contributions of history-taking, physical examination, and laboratory investigation to diagnosis and management of medical outpatients. Br Med J 1975;2:486-9.

2. Heneghan C, Glasziou P, Thompson M, et al. Diagnostic strategies used in primary care. BMJ 2009;338:b946.

3. Jones R, Barraclough K, Dowrick C. When no diagnostic label is applied. BMJ 2010;340:c2683.

4. Donner-Banzhoff N, Seidel J, Sikeler AM, et al. The phenomenology of the diagnostic process: a primary care-based survey. Med Decis Making 2017;37:27-34.

5. Feufel MA. Bounded Rationality in the Emergency Department. 2009:332.
6. MAXQDA. Berlin: VERBI Software. Consult. Sozialforschung GmbH 2010.

7. Elstein AS, Schulman LS, Sprafka SA. Medical problem-solving: an analysis of clinical reasoning. Cambridge (MA: Harvard University Press, 1978:330.

8. Norman G, Barraclough $\mathrm{K}$, Dolovich L, et al. Iterative diagnosis. BMJ 2009;339:b3490.

9. Donner-Banzhoff N, Hertwig R. Inductive foraging: improving the diagnostic yield of primary care consultations. Eur J Gen Pract 2014;20.

10. Holsti OR. Content analysis for the social sciences and content analysis for the social sciences and humanities. Reading, MA: Addison-Wesley, 1969.

11. Mayring P. Qualitative Inhaltsanalyse: Grundlagen und Techniken. 12 edn. Weinheim: Beltz, 2015.

12. Stolper E, van Royen P, Dinant GJ. The 'sense of alarm' ('gut feeling') in clinical practice. A survey among European general practitioners on recognition and expression. Eur J Gen Pract 2010;16:72-4.

13. Gigerenzer G. Todd PM, and the ABCRG. Simple heuristics that make us smart. New York: Oxford University Press, 1999.

14. Todd PM, Gigerenzer G. Ecological rationality: Intelligence in the world. Oxford, New York: Oxford University Press, 2012.

15. Feufel MA. How to uncover sources of unwarranted practice variation: a case study in emergency medicine. Qual Health Res 2018;28:1486-98.

16. Lippa KD, Feufel MA, Robinson FE, et al. Navigating the decision space: shared medical decision making as distributed cognition. Qual Health Res 2017;27:1035-48.

17. Chmiel C. Walk-ins seeking treatment at an emergency department or general practitioner out-of-hours service: a cross-sectional comparison: BioMed Central Ltd, 2011.

18. Thompson MI, Lasserson D, McCann L, et al. Suitability of emergency department attenders to be assessed in primary care: survey of general practitioner agreement in a random sample of triage records analysed in a service evaluation project. BMJ Open 2013;3:e003612.

19. O'Keeffe N. The effect of a new general practice out-of-hours cooperative on a county hospital accident and emergency department. Ir J Med Sci 2008;177:367-70. 